\title{
NORMALIZATION OF BRAIN ACTIVATIONS IN DEPRESSION, WITH A SUBJECT-INDEPENDENT PATTERN-BASED BRAIN-COMPUTER INTERFACE SYSTEM.
}

\author{
Jaime Pereira1,2, Andreas Ray³, Mohit Rana ${ }^{3}$, Patricia Opazo², Claudia Brett² ,Ishani Takar¹, Rafael Torres², \\ Ranganatha Sitaram ${ }^{* 1,2,4}$, Sergio Ruiz ${ }^{* 1,2}$ \\ 1. Laboratory for Brain-Machine Interfaces and Neuromodulation, Pontificia Universidad Católica de Chile, Santiago, Chile. 2. Department of \\ Psychiatry and Neuroscience Division, Pontificia Universidad Católica de Chile, Santiago, Chile, 3. Institute of Medical Psychology and \\ Behavioral Neurobiology, University of Tübingen, Tübingen, Germany. 4. Institute for Biological and Medical Engineering, Pontificia \\ Universidad Católica de Chile, Santiago, Chile \\ *sruiz@uc.cl / rasitaram@uc.cl
}

\section{Introduction}

Most neurofeedback studies have been built upon the idea that a neurological or psychiatric patient can learn by feedback training to produce brain activity closer to the healthy activation levels for the purpose of alleviating their symptoms. However, due to the inherent altered brain functioning, patients could have difficulties in achieving healthy pattern of brain signals. To overcome this difficulty, we aimed to develop a neurofeedback system that could potentially retune the brain activity of patients to progressively shift towards healthy pattern of activity. Based on this paradigm, we designed the present study with two major aims: 1) to train depressive patients to achieve a healthy emotional brain-state, aided by a $\mathrm{BCl}$ system coupled with a "subject-independent (or population-based) classifier" of emotional states, created from EEG data of healthy individuals, and 2) to explore the use of this system as a potential clinical and neuroscientific tool.

\section{Materials and Methods}

A subject-independent pattern-based classifier (SIC) of brain states (based on the Common Spatial Patterns (CSP) feature extraction of EEG signals, and Support Vector Machine, SVM) was created, with information coming from 19 healthy adult females during happiness self-induction (and motor imagery) derived from data collected in a previous study (Rey et al, 2005). This classifier was coupled to a $\mathrm{BCl}$ system (using a 32-channel system covering the whole skull) to train 4 female patients (Mean age: 27 y.o.) with mild to moderate depressive symptoms to "match" healthy brain state of positive emotion.
Patients participated in 6 days of self-regulation training for 3 weeks. Each day consisted of 4 training runs composed of 8 blocks (20s. each block) of brain-self regulation (4 blocks during positive emotional imagery followed by 4 blocks of motor imagery). A baseline measurement of EEG resting state signal was collected for 10 seconds at the beginning and the middle of each run-in order to use the signals for classifier bias correction. Self-regulation blocks were visually guided by the result of the SVM classifier to receive the information of the similarity of the patients's brain state compared with the one provided by the SIC in real-time (updated every 1 second). Clinical tests (blinded) were collected before and after the training.

\section{Results and Conclusions}

2 Participants were able to reproduce the positive brain patterns of the original group of healthy subjects to variable extents, as reflected in the classification accuracies of the SIC (Figure 1 for two representative patient data). Mood modifications towards the alleviation of depressive symptoms were self-reported for all participants (BDI Pre - Post training: M: 7.25; R: 1 to 12) and evaluated by a blinded clinician (HDS Pre - Post training: M: 7.75; R: -1 to 15 ).
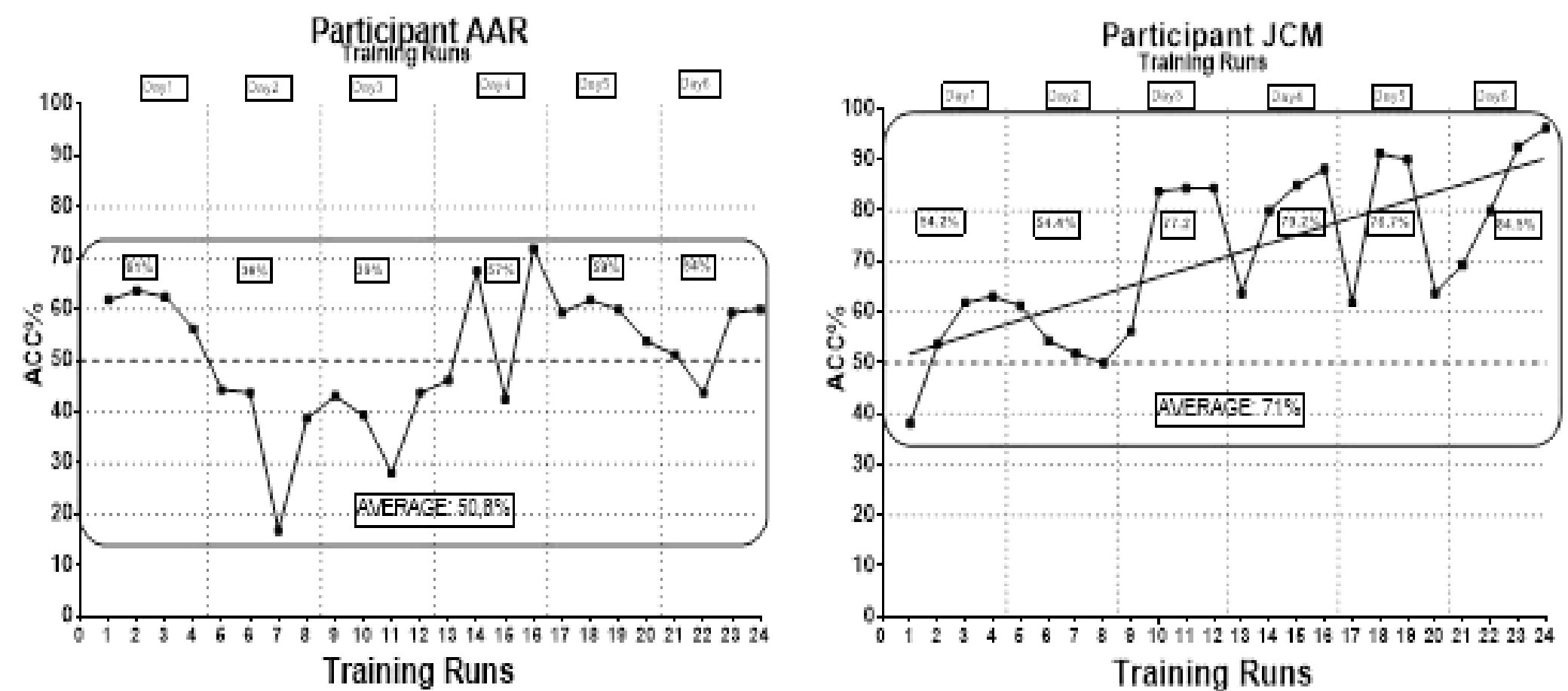

Figure 1. Classification accuracy throughout the training of two representative subjects. The data samples are plotted on the $x$-axis against the SVM values (accuracy percentage) on the $y$-axis during 6 days of training. The average of the accuracy percentage per day and of all training are placed inside black frames. Significant Linear regression between Run progression and accuracy percentage was observed and plotted (slope 1,6 $\pm .34 ;$ r2: 0,52 p: <.0001).

These results represent progress towards the development of automatic recognition of brain states and its real-time feedback as a potential clinical and neuroscientific tool. This study is ongoing, and the final aim to increase the sample and to incorporate a control group of participants without contingent feedback from SIC.

References: [1] Ray, A.M. (2015), Front. Behav. Neurosci., vol. 9, pp. 269. [2] Sitaram, R. (2017), Nat Rev Neurosci., vol. 18, no. 2, pp. 86100. [3] Ruiz, S. (2013), Hum Brain Mapp., vol. 34, no. 1,pp. 200-12. [4] Linden, D.E. (2012), PLoS One, vol. 7, no. 6, pp. e38115. 